\title{
Crottet, Grièges, Replonges, Saint-André-de-Bâgé
}

\section{Cécile Ramponi}

\section{OpenEdition \\ Journals}

Édition électronique

URL : http://journals.openedition.org/adlfi/1831

ISSN : 2114-0502

Éditeur

Ministère de la culture

Référence électronique

Cécile Ramponi, "Crottet, Grièges, Replonges, Saint-André-de-Bâgé », ADLFI. Archéologie de la France Informations [En ligne], Rhône-Alpes, mis en ligne le 01 mars 2008, consulté le 19 avril 2019. URL

http://journals.openedition.org/adlfi/1831

Ce document a été généré automatiquement le 19 avril 2019

(C) Ministère de la Culture et de la Communication, CNRS 


\title{
Crottet, Grièges, Replonges, Saint- André-de-Bâgé
}

\author{
Cécile Ramponi
}

\section{Identifiant de l'opération archéologique : 9700}

Date de l'opération : 2008 (EX)

1 Une campagne de diagnostic archéologique sur le tracé du contournement sud de Macon (future autoroute A 406) a été menée sur une superficie de presque 65 ha, sur les communes de Saint-André-de-Bâgé, Crottet, Replonges et Grièges. Ce tracé recoupe deux unités géologiques principales, le plateau bressan dans les deux tiers nord et les terrasses alluviales de la Saône au sud.

2 Au total, 1558 sondages ont été réalisés, représentant $6,88 \%$ de la surface à ouvrir. Des vestiges mobilier et immobilier du Néolithique final, du Bronze final, du Hallstatt C, de La Tène ancienne, de La Tène finale et du Haut-Empire, ont été mis au jour.

\section{La Préhistoire}

3 Aucune structure préhistorique n'est avérée sur le tracé. Les seuls indices remontant à cette période sont des silex taillés. Sur la partie nord du tracé, Zones A à E, certains des éléments lithiques ne dépareraient pas dans des assemblages du Néolithique final. En zone $\mathrm{F}$, et surtout de part et d'autre de la Veyle (zones $\mathrm{G}$ et $\mathrm{H}$ ), ce matériel signale une occupation plus récente, probablement au cours du Campaniforme. Une petite concentration de galets thermofractés lui est associée en zone $\mathrm{H}$. A noter que pour les attributions chronologiques, ce mobilier lithique n'est accompagné d'aucune céramique permettant une datation fine de la culture matérielle.

4 Des dates ${ }^{14} \mathrm{C}$ entreprises sur des bois devraient permettre de poser des jalons chronologiques dans la compréhension de la formation de la rive gauche de la Saône. 


\section{La Protohistoire}

5 Pour l'âge du Bronze les témoins retrouvés remontent uniquement à la fin de cette période.

6 En zone $\mathrm{D}$ et $\mathrm{H}$, deux dépôts de crémation en urne ont été prélevés. Les céramiques contenant les ossements remontent à l'extrême fin du Bronze final. Ces dépôts distants de $980 \mathrm{~m}$ paraissent isolés, même si sur la zone D un lambeau de paléosol, contenant du mobilier protohistorique, a été préservé à la faveur d'une dépression.

7 L'indice de site le plus probant se trouve en zone G, au sud-ouest de la voie ferrée, avec au moins deux fosses.

8 Le mobilier retrouvé pourrait faire remonter cette occupation à la phase moyenne du Bronze final, le Bronze final 2. Nous ne pouvons caractériser l'installation, mais, la présence systématique de mobilier céramique protohistorique dans les sondages alentours plaide en la faveur d'un site.

9 Pour l'âge du Fer, deux indices d'installations sont à prendre en compte. En zone C, a été mise au jour une fosse, apparemment isolée, que le mobilier permet de rattacher au tout début du $1^{\text {er }}$ âge du Fer, le Hallstatt $C$. Les sondages alentours n'ont pas livré d'indices contemporains. Nous sommes peut-être en marge d'une implantation plus importante à moins qu'il ne s'agisse d'une installation temporaire. En revanche, la Zone A a permis de mettre en évidence un horizon protohistorique matérialisé, entre autre, par un niveau de tessons d'amphore de Marseille et la présence d'une structure en creux. Le type d'amphore retrouvée est produit de la fin $d u \mathrm{VI}^{\mathrm{e}} \mathrm{s}$. au début du $\mathrm{IV}^{\mathrm{e}}$ av. J.-C. Nous proposons donc d'inscrire l'installation dans cette fourchette chronologique, du début du $2^{\mathrm{e}}$ âge du Fer. La nature, l'étendue et la fonction de cette installation restent indéterminées. Il faut insister sur le fait que les sites de cette période révélant des importations, sont rares dans la région. Autre fait remarquable, la présence sur la zone $\mathrm{G}$, en position secondaire, de petits fragments d'amphore de Marseille. Ces points de découvertes sont distants de $3 \mathrm{~km}$ et ne peuvent être interprétés comme la même occupation.

10 Hormis ces installations avérées, quelques structures isolées ont été associées à la Protohistoire : en zone B, un probable foyer, une fosse en zone $\mathrm{E}$ et un foyer ou structure de combustion à galets dans la partie nord-est de la zone G. Du mobilier céramique résiduel, attribué à la Protohistoire de manière large, car n'étant pas tourné, saupoudre le tracé. Les quantités retrouvées sont variables mais souvent en relation avec des installations avérées. Ainsi, c'est dans la partie occidentale de la zone $G$, au-dessus et autour des structures de la fin de l'âge du Bronze, que le plus grand nombre de fragments a été mis au jour. Vient ensuite la zone $C$, à proximité de la fosse du Hallstatt $C$ et de l'installation de La Tène finale. L'absence de formes, de céramiques fines et le fractionnement important des éléments ne permet pas de trancher quand à leur rattachement à l'une ou l'autre de ces cultures. Enfin en zone $\mathrm{D}$, de la céramique a été conservée dans le paléosol situé à un peu plus d'une centaine de mètres du dépôt de crémation en urne du Bronze final 3.

11 Pour terminer avec l'âge du Fer, il faut attirer l'attention sur la présence, en très petite quantité, de fragments d'amphore Dressel 1, sur presque toutes les zones du tracé, sans 
pour autant qu'elle soit systématiquement associée à du mobilier antique. Par ailleurs, en Zone $\mathrm{C}$, un fossé pourrait remonter à La Tène finale, mais sans certitude.

\section{La période gallo-romaine}

12 Les installations de la période gallo-romaine, autant par leur nature que par leur mobilier, reflètent un caractère profondément rural.

13 En Zone $\mathrm{C}$, a été mise en évidence une occupation dont l'implantation remonte à la fin du second âge du Fer (La Tène finale) et qui perdure au Haut-Empire. Elle se compose de dépressions et de fossés. Au moins deux de ces dépressions, s'apparentent à des mares par la nature de leur comblement et, dans plusieurs sondages, des niveaux contenant des tuiles et du mobilier de La Tène finale et de l'époque gallo-romaine ont été observés. La durée de vie et les remaniements opérés dans les implantations rendent sa compréhension difficile en sondage. Il s'agit de l'implantation la plus importante retrouvée sur le tracé.

14 On retrouve en zone $\mathrm{F}$, une probable mare isolée et à $120 \mathrm{~m}$ d' elle, des petites fosses de fonction indéterminée et deux fossés de la période gallo-romaine. Ces dernières structures traduisent des activités rurales certainement situées très en marge d'une véritable installation pérenne de type habitat.

15 Sans lien avec ces aménagements, tout au long du tracé, quelques structures isolées ont pu être rattachées à la période gallo-romaine de manière large. Signalons en Zone A, un épandage de tuiles; dans le secteur central de la Zone C, de larges dépressions comblées naturellement mais contenant un peu de mobilier gallo-romain ainsi que deux petits fossés; en Zone D, une grosse fosse présentant un peu de mobilier et en Zone E, un probable gros fossé.

16 Au final, même si un peu de mobilier gallo-romain résiduel a été retrouvé hors structure, la présence dans le même temps de fragments d'amphores italiques dresse le portrait d'un terroir exploité de manière extensive dès la fin de l'époque gauloise ou tout début de la Conquête et au moins jusqu'à la fin du Haut-Empire.

\section{Les périodes médiévale et moderne}

17 Ces périodes, malgré les hectares parcourus et les espérances soulevées par l'étude documentaire (Barthélémy, Vérot-Bourrely, Goy, 1998), sont très peu représentées. Elles se résument en quatre points de découverte de céramiques. Ces tessons, retrouvés sur les communes de Crottet et de Grièges, comptent un total général de 27 éléments.

18 A noter que la majorité de ces céramiques sont localisées dans deux sondages voisins, et un troisième situés à moins de $100 \mathrm{~m}$, sur la commune de Crottet. Ces lots, hors structures, paraissent mélangés avec aussi bien des éléments du Moyen Age central ( $\mathrm{X}^{\mathrm{e}} \mathrm{s}$. -XII s.), de la fin du Moyen Age (XV' ${ }^{e}$ s. - $\mathrm{XVI}^{\mathrm{e}}$ s.), de l'époque moderne (XVII ${ }^{\mathrm{e}}$ s. -XVIII ${ }^{\mathrm{e}} \mathrm{s}$.) et de l'époque contemporaine (XIX ${ }^{\mathrm{e}} \mathrm{s}$.).

19 Ces sondages sont distribués de part et d'autre d'un axe de circulation, la RD 933, mais surtout proches du hameau de Villeneuve situé à $600 \mathrm{~m}$ vers le sud. Ce hameau est attesté au moins depuis 1757 (Goy, dans Barthélémy, Vérot-Bourrely, Goy, 1998, p. 12). 
20 Les quelques tessons modernes (XVII ${ }^{\mathrm{e}}$ s.-début XVIII ${ }^{\mathrm{e}} \mathrm{s}$.) retrouvés sur la commune de Grièges sont associés à des bandes gravillonneuses pouvant être interprétées, avec circonspection, comme un reste de chemin ou comme un apport de matériaux dans le but d'assainir le terrain. Aucun autre témoin de cette période n'a été trouvé dans le secteur, si ce n'est de nombreuses fosses charbonneuses, probables restes de brûlis, dont la datation est incertaine mais peu ancienne, probablement contemporaine.

21 L'apport de cette découverte indique vraisemblablement une utilisation de ces terrains (pâtures ou champ) depuis au moins le XVII ${ }^{\mathrm{e}} \mathrm{s}$. On peut même faire remonter la connaissance dans les archives de l'utilisation des terrains proches de la Saône comme pâturages au $\mathrm{XVI}^{\mathrm{e}}$ s., plus particulièrement au lieu-dit Plagne, comme l'indique une mention de 1522 (Goy, dans Barthélémy, Vérot-Bourrely, Goy 1998, p. 16).

22 Sur cette dernière commune, il est logique de ne pas trouver d'occupation fixe en raison des fréquents débordements de la Saône. L'étude documentaire préalable au projet (Goy, dans Barthélémy, Vérot-Bourrely, Goy 1998, p. 19-23) a montré que les inondations sont mentionnées depuis le XII ${ }^{\mathrm{e}} \mathrm{s}$.

\section{INDEX}

\section{operation Expertise (EX)}

Index chronologique : âge du Bronze, âge du Fer, Antiquité, Haut-Empire, Moyen Âge, Néolithique final

Thèmes : amphore italique, amphore massaliète, céramique campanienne, dépôt funéraire, galet, incinération, ossement humain, tuile, urne

Index géographique : Rhône-Alpes, Ain (01), Crottet

\section{AUTEUR}

CÉCILE RAMPONI

INRAP 